\title{
Complexity in the sunspot cycle
}

\author{
G. Consolini ${ }^{1}$, R. Tozzi ${ }^{2}$, and P. De Michelis ${ }^{2}$
}

1 INAF - Istituto di Fisica dello Spazio Interplanetario, 00133 Roma, Italy

e-mail: consolini@ifsi-roma.inaf.it

2 Istituto Nazionale di Geofisica e Vulcanologia, 00143 Roma, Italy

Received 2 October 2008 / Accepted 5 June 2009

\section{ABSTRACT}

\begin{abstract}
The occurrence of complexity in the solar cycle, as monitored by the sunspot area butterfly diagram, is investigated by means of the natural orthogonal composition (NOC) technique and information theory approach. Although the butterfly diagram may be reconstructed using only two modes as already found in other papers for the Hale cycle, on deeper investigation it is possible to notice that the high variability, complexity, and stochasticity observed during the solar cycle are missing. A full description of the complex evolution of the solar cycle requires at least 30 modes. We show that these modes identify two different dynamical regimes, whose existence is also confirmed by the analysis of the Lyapunov exponents of the associated principal components. We suggest that the existence of these two physical dynamical regimes is at the origin of the dynamical complexity of the solar cycle. We attempt a discussion of these dynamical regimes also in terms of a nearly stable dynamo process described by the first two modes and a local superficial turbulent dynamo responsible for the more stochastic features observed in the solar cycle.
\end{abstract}

Key words. Sun: activity - Sun: sunspots - methods: statistical - chaos

\section{Introduction}

The Sun has been observed through telescopes for almost four centuries. During this period, our understanding of the Sun and of its dynamics has undergone a profound revolution. However, it is only in the past few decades that the traditional view of many solar features has been completely reconsidered in the light of both theoretical advances and high-resolution ground and space observations.

Solar activity is traditionally estimated by the relative sunspot number $R$, also known as the Wolf sunspot number, which represents a qualitatively reliable proxy of the activity itself. The Wolf sunspot number $R$ is important because it is one of the oldest and longest running direct record of solar activity, with reliable observations starting in 1610 .

However, a more informative characterization of the solar cycle in terms of sunspots is provided by the spatio-temporal diagram built by plotting, for each solar rotation cycle (Carrington cycle), the total area of observed sunspots as a function of latitude. This diagram, known as butterfly diagram, shows that sunspots are not randomly distributed over the solar surface but, at any stage of the cycle, they are concentrated in a latitudinal band across the solar equator. Since sunspots represent a surface manifestation of the emergence of the toroidal magnetic field residing in the solar interior, the butterfly diagram also represents a spatio-temporal map of the solar internal large-scale toroidal magnetic field.

In spite of its nearly-cyclic nature, the solar cycle is characterized by amplitude, period, and shape, which vary irregularly. These irregularities appear to be an intrinsic feature of the solar cycle being observed in many other solar observables including irradiance, surface flows, and polar faculae counts. Anyway, the origin of these irregularities is still unclear.

Using the temporal and latitudinal distribution of sunspots recorded since 1874, it was proposed (Mininni et al. 2002) that the solar magnetic cycle, investigated by means of the butterfly diagram, might be interpreted as being the result of the superposition of two oscillations, characterized by constant amplitude and phase and by a period close to 22 years, on a stochastic background. This suggested that the spatio-temporal irregularities observed in the solar magnetic cycle were not a plain manifestation of low-dimensional chaos being due to the interaction of two superposed antisymmetric modes with a stochastic background (Mininni et al. 2002, 2004). Similar results were found by Lawrence et al. (2005), who investigated the 22 year solar cycle by NSO Kitt Peak synoptic maps, and by Vecchio et al. (2005), who studied the spatio-temporal features of 11-year solar activity using the green coronal emission line at $530.3 \mathrm{~nm}$. In contrast, Letellier et al. (2006) showed that the 22-year solar magnetic cycle, as reconstructed by sunspot time series, is in agreement with a low-dimensional chaotic dynamics. In detail, they showed that the phase-space diagram (the phase portrait) of the sunspot number resembles that of a Rössler dynamical system.

The aforementioned results seem to point towards the occurrence of spatio-temporal and/or dynamical complexity in the solar cycle. Quoting Nicolis \& Nicolis (2007), "complexity is not a mere metaphor or a nice way to put certain intriguing things, it is a phenomenon that is deeply rooted into the laws of nature, where systems involving large numbers of interacting subunits are ubiquitous". Furthermore, Chang et al. (2006) defined the dynamical complexity as "a phenomenon exhibited by a nonlinearly interacting dynamical system within which multitudes of different sizes of large scale coherent structures are formed, resulting in a global nonlinear stochastic behavior for the dynamical systems, which is vastly different from that could be surmised from the original dynamical equations". In this framework, complexity can be defined as the tendency of a non-equilibrium system to show a certain degree of spatio-temporally coherent features resulting from the competition of different basic spatial 
patterns (Badii \& Politi 1997) playing the role of interacting subunits. As a result, the spatio-temporal evolution of these complex systems may display evolutionary events as for instance those observed in the case of turbulent systems. It is important to emphasize that complexity requires the occurrence of nonlinearities and the intertwining of order and disorder (Nicolis \& Nicolis 2007), and that it is generally related to the emergence of selforganization in open systems (Klimontovich 1991, 1996).

Here, using the natural orthogonal composition (NOC) technique, we investigate the emergence of spatio-temporal complexity in the 11-year solar cycle, as monitored by the sunspot area butterfly diagram. By applying a 2nd order complexity measure (Shiner et al. 1999), we show that spatio-temporal and/or dynamical complexity is an intrinsic property of the solar cycle, and that the long-term evolution of solar cycle activity seems to point towards a more regular (less complex) behavior.

The paper is organized as follows. Section 2 provides a brief introduction to the NOC technique. In Sect. 3, we describe the data set and results from the NOC analysis, the study of the chaotic and spectral features of the principal components (PCs). In particular, we pay attention to the first two PCs by comparing their phase portrait with that coming from a stochastic version of a Lotka-Volterra predator-prey model. In this section, we also show the occurrence of dynamical complexity obtained by the application of information theory descriptors. In Sect. 4, we summarize our findings and draw conclusions.

\section{The natural orthogonal composition technique: a brief introduction}

The natural orthogonal composition (NOC) (Jackson et al. 1991; Golovkov et al. 1992) is a technique that can be used for feature extraction, i.e., given a set of multivariate measurements NOC is expected to be able to provide a smaller set of uncorrelated variables or components whose proper combination gives back the larger starting set. In practice, given a set of observations, it is possible to estimate a set of independent eigenvectors and eigenvalues whose combination allows rewriting the observed variables. Since this operation corresponds to transforming the natural basis on which we observe the variable, into a new orthogonal basis by diagonalizing the variance matrix, the NOC technique consists essentially of a rotation of the old basis into the new one.

NOC has been widely used in literature, for instance for the study of daily magnetic variations (Golovkov et al., 1978; Xu \& Kamide, 2004), for space-time analysis of the main geomagnetic field (Rotanova et al., 1982), for the study of global models of the geomagnetic field (Xu, 2003), and even for the automatic calculation of geomagnetic $\mathrm{K}$ indices (Golovkov et al. 1989). Before describing the application of NOC, we introduce the method briefly.

We assume that we measure a spatio-temporal variable $\psi(x, t)$ at a certain time $t$ consisting of $m$ elements, where $m$ is the number of latitudinal spatial positions (pixels). Given a number of samples at different spatio-temporal locations, the application of NOC allows us to extract a smaller set of variables, such as empirical orthogonal functions (EOFs) and principal components (PCs), capable of describing the entire set of observations. Although there are many methods capable of doing this job, the benefit of NOC is that the set of functions (EOFs) used in the expansion of the time series is not determined in advance but is estimated using only the observed data.
We now write the spatio-temporal field $\psi\left(x_{i}, t_{j}\right)$ at position $x_{i}$ and time $t_{j}$ to be:

$\psi\left(x_{i}, t_{j}\right)=\sum_{k=1}^{N} A^{k}\left(t_{j}\right) \phi^{k}\left(x_{i}\right)$,

where the collection of values $\psi\left(x_{i}, t_{j}\right)$ provides the elements $\psi_{i j}$ of the $m \times n$ matrix $\hat{\Psi}$ with rows corresponding to the observations made at a fixed spatial position $x$ and at $n$ values of time, and columns to the observations made at $m$ positions $x$ and at a certain time $t$, and $N$ is the number of components chosen for the decomposition (i.e., the truncation level). In Eq. (1), the EOF is $\phi^{k}(x)$, which is the mode of the $k$ th component with elements $\phi_{i}^{k}(i=1,2, \ldots, m)$ describing the spatial distribution (i.e., it is the basis used for the expansion), and the PC is $A^{k}(t)$, which is the amplitude of the corresponding mode with elements $a_{j}^{k}$ for $(j=1,2, \ldots, n)$. In practice, the EOFs $\phi^{k}(x)$ and the PCs $A^{k}(t)$ are capable of identifying the space-dependent and the timedependent behavior of the spatio-temporal field $\psi(x, t)$.

To evaluate the EOFs $\phi^{k}$ and the associated PCs $A^{k}$ from a data set, it is necessary to minimize the error made in the representation by means of the expansion of Eq. (1) of observed data $\psi_{i j}$, this error $\delta$ can be defined as the sum over all $i$ s and $j$ s of the squared differences between observed and estimated data, given by

$\delta=\sum_{i=1}^{m} \sum_{j=1}^{n}\left[\psi_{i j}-\sum_{k=1}^{N} a_{j}^{k} \phi_{i}^{k}\right]^{2}$.

Since we are looking for a number of components able to describe completely the observed variable, one additional assumption is that the EOFs $\phi^{k}$ are orthogonal and the PCs $A^{k}$ vary independently. Based on these assumptions the minimization process reduces to solving an eigenvalue/eigenvector problem (secular equation)

$\hat{V} \boldsymbol{\phi}^{k}=\lambda_{k} \boldsymbol{\phi}^{k}$,

where $\hat{V}$ is the $N \times N$ covariance matrix with elements $v_{i j}$ given by

$\hat{V}=\hat{\Psi}^{T} \hat{\Psi}$.

Here $\hat{\Psi}$ is, as already mentioned, the matrix whose elements $\psi_{i j}$ are the values of the observed variable $\psi\left(x_{i}, t_{j}\right)$. Once we obtain the EOFs $\phi^{k}$, the PCs will be evaluated by simply projecting the data along each EOFs,

$\boldsymbol{A}^{k}=\hat{\Psi} \phi^{k}$.

From Eq. (3), which is the well-known secular equation, it is possible to estimate the eigenvalues $\lambda^{k}$ and their corresponding eigenvectors $\phi^{k}$ for $k=1, \ldots, N$, and then the amplitudes $A^{k}$. We note that the eigenvalues $\lambda_{k}$ provide a measure of the root-meansquare value of the corresponding PC, i.e., $\lambda_{k}=N\left\langle y_{k}^{2}\right\rangle$, where $\left\langle y_{k}^{2}\right\rangle$ is the mean-square value of the $k^{\text {th }} \mathrm{PC}$. Furthermore, once the eigenvalue spectrum $\lambda_{k}$ has been evaluated, it is possible to establish the number $N^{*}<N$ of fundamental EOFs that are sufficient to capture most of the properties of the observed variable, i.e.,

$\psi(x, t) \sim \sum_{k=1}^{N^{*}} A^{k}(t) \phi^{k}(x)$. 


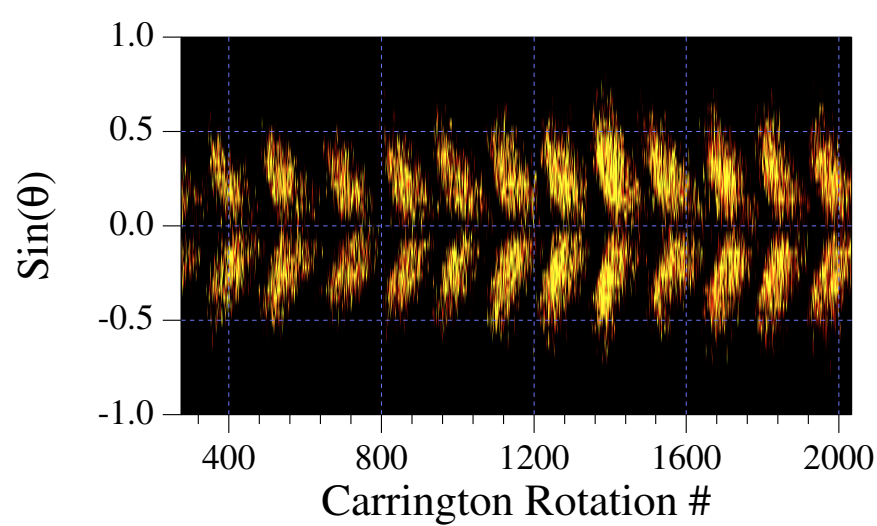

Fig. 1. The sunspot area butterfly diagram from RGO/USAF/NOAA starting on May, 1874. The color (black-red-yellow) is proportional to the sunspot area that is plotted as a function of time (Carrington rotation number \#) and latitude.

We note that the NOC technique is strictly valid for spatiotemporal signals caused by the linear superposition of independent modes. Consequently, the interpretation of results of its application to spatio-temporal signals produced by nonlinear processes could be either difficult or doubtful owing to the approximation of the nonlinear interaction of independent modes with their linear combination.

\section{Data description and results}

\subsection{Data and NOC analysis}

To investigate the occurrence of spatio-temporal complexity in the 11-year solar activity cycle, we consider the total sunspot area butterfly diagram (see Fig. 1), compiled from Royal Greenwich Observatory and USAF Solar Optical Observing Network observations and available at the NASA Solar Physics Marshall Space Flight Center ${ }^{1}$. The butterfly diagram is, indeed, the only spatio-temporal dataset that, spanning over more than 12 solar cycles, can be considered sufficiently long to apply the NOC analysis, which requires a set of temporal snapshots (here 1759 Carrington rotations) larger in number than the total number of spatial observations (here 50 latitudinal bins). The butterfly diagram $\psi(x, t)$ refers to the latitudinal $(x)$ and temporal $(t)$ distribution of the sunspot areas in units of millionths of a hemisphere since May, 1874. Data, representing the total sunspot area, are organized in 50 latitude bins per Carrington rotation ( $\sim 27.28$ days). The latitude bin locations are uniformly distributed with respect to $\sin (\theta)$, where $\theta$ is the latitude (i.e., $x_{i}=\sin \left(\theta_{i}\right)$ ), while the temporal window spreads over 1759 Carrington rotations. The sunspot butterfly diagram data used in this work was compiled following RGO/USAF/NOAA instructions $^{1}$ for data after 1976 , which requires to increase USAF/NOAA spot areas by a factor of 1.4.

According to the previous section describing the NOC technique, to find the EOFs and their associated PCs, it is necessary to solve the secular equation of Eq. (3), where $\hat{V}$ is the covariance matrix, compiled from the spatio-temporal dataset, which is a $50 \times 50$ matrix (of 50 latitudinal bins) with elements given by $v_{i j}=\sum_{k} \psi\left(\sin \left(\theta_{i}\right), t_{k}\right) \psi\left(\sin \left(\theta_{j}\right), t_{k}\right)$. All calculations were performed by utilizing user-defined macros within a commercially available data analysis software (Igor Pro, WaveMetrics).

Figure 2 shows the eigenvalue $\lambda_{k}$ spectrum evaluated from the secular equation of Eq. (3). Two different physical regimes

\footnotetext{
${ }^{1}$ http://Science.nasa.gov/ssl/pad/solar/greenwch.htm
}

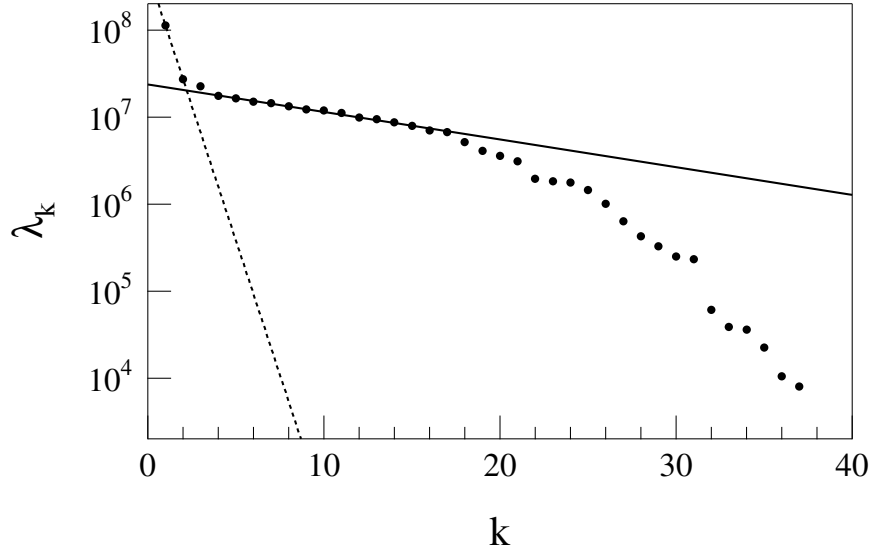

Fig. 2. The eigenvalue $\lambda_{k}$ spectrum for $k \in[1,40]$ as evaluated using the NOC technique. Dotted and solid lines in the inset are exponential fits for $k \leq 2$ and $k \in[3,18]$, respectively.

are identifiable for $k$ below and above $k=3$. In particular, the first eigenvalue is larger by 4 times than the second, and 5 times than the third. The other eigenvalues monotonically decrease for increasing $k$, exhibiting a cut-off for $k>18$. Because this spectrum can be considered as the equivalent of an energy spectrum, and is associated with the variance of the various components, we can assume that most of the variability observed in the solar cycle can be explained in terms of a very small number of components $(n \leq 3)$.

Figures 3 and 4 show the first six EOFs and the corresponding PCs. These functions provide, respectively, the latitudinal dependence $\left(\mathrm{EOF} \rightarrow \phi^{k}(\theta)\right)$ and the time variation $\left(\mathrm{PC} \rightarrow A^{k}(t)\right)$ in the modes, ordered according to the eigenvalue spectrum $\lambda_{k}$. Looking at the structures of the EOFs, one can immediately note the inherent symmetry of these EOFs. This is particularly evident for the EOFs with $k=1,2$, which are symmetric with respect to the equator (even-parity), thus resembling the symmetry observed in the butterfly diagram. In contrast, the EOF with $k=3$ is antisymmetric (odd-parity) with respect to the equator. The symmetry features are less evident with increasing $k$ and may take into account the so-called north-south asymmetries (Sokoloff \& Nesme-Ribes 1994). We note that, although all of the features of the PCs seem to be characterized by a nearly cyclic behavior resembling the solar activity cycle, they have different intermittency degrees. We find that the kurtosis excess $\kappa$, which indicates the deviation from Gaussianity, is $\kappa \sim 3$ and $\kappa>7$ for the first two PCs and for the remainder, respectively.

To characterize the cyclic behavior of the PCs and find evidence of intrinsic periodicities, we evaluate the Fourier power spectra of the $A^{k}(t)$ for the first six modes. These spectra are shown in Fig. 5, where it is possible to note that the periodic structure of the solar cycle is due mainly to only the first two EOFs and PCs. As a matter of fact, only the spectra of the first two PCs display a distinctive periodicity at $T_{1} \sim 11$ (10.9) yr. A secondary, less apparent periodicity $\left(T_{2} \sim 5.5 \mathrm{yr}\right)$ is found in correspondence of the second harmonic $\left(T_{2} \sim T_{1} / 2\right)$. None of the spectra of the other PCs exhibit any clear periodicity. We remark that, while the first periodicity agrees with that found by the previous analyses of the solar magnetic cycle (Mininni et al. 2002, 2004; Lawrence et al. 2005), the periodicity at $T_{2}$ is detected only by studying the 11-year cycle. Furthermore, the 7 -year periodicity found by Mininni et al. $(2002,2004)$ is not evident in our analysis. 


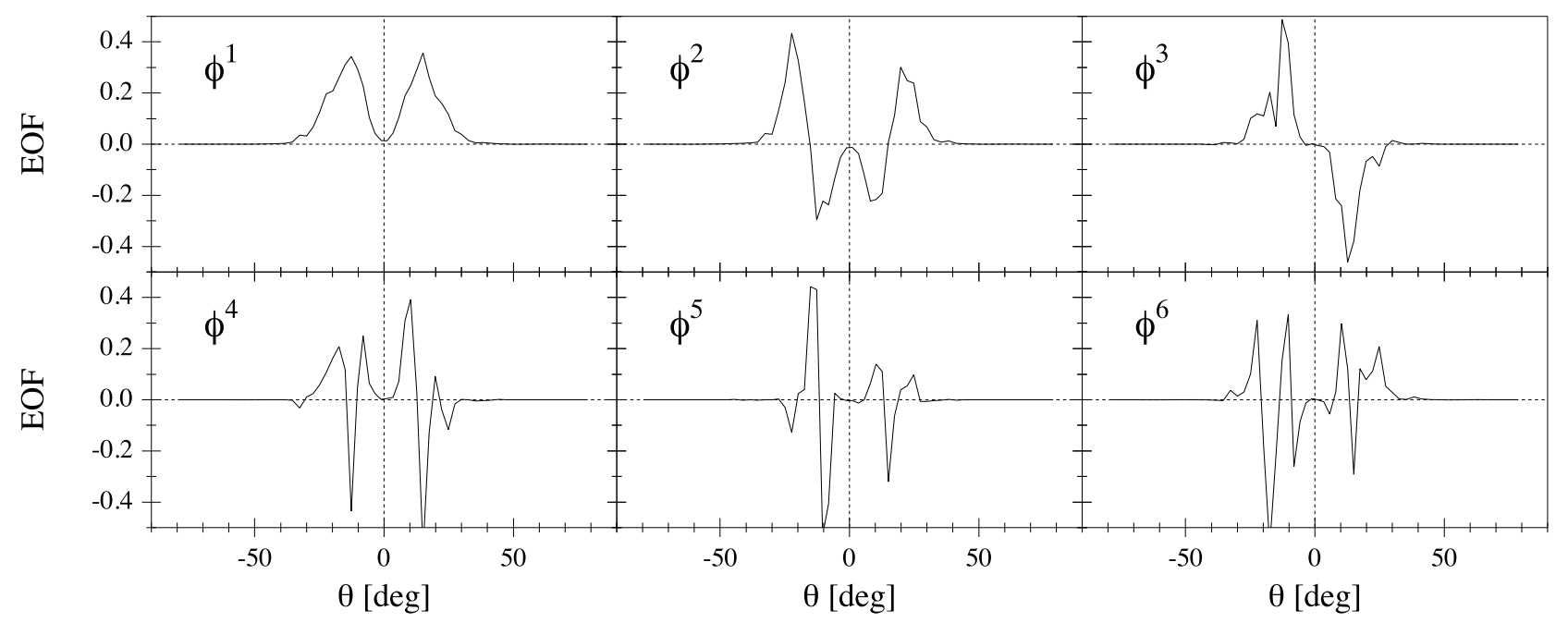

Fig. 3. The first six empirical orthogonal functions (EOFs) ordered according to the eigenvalue spectrum.

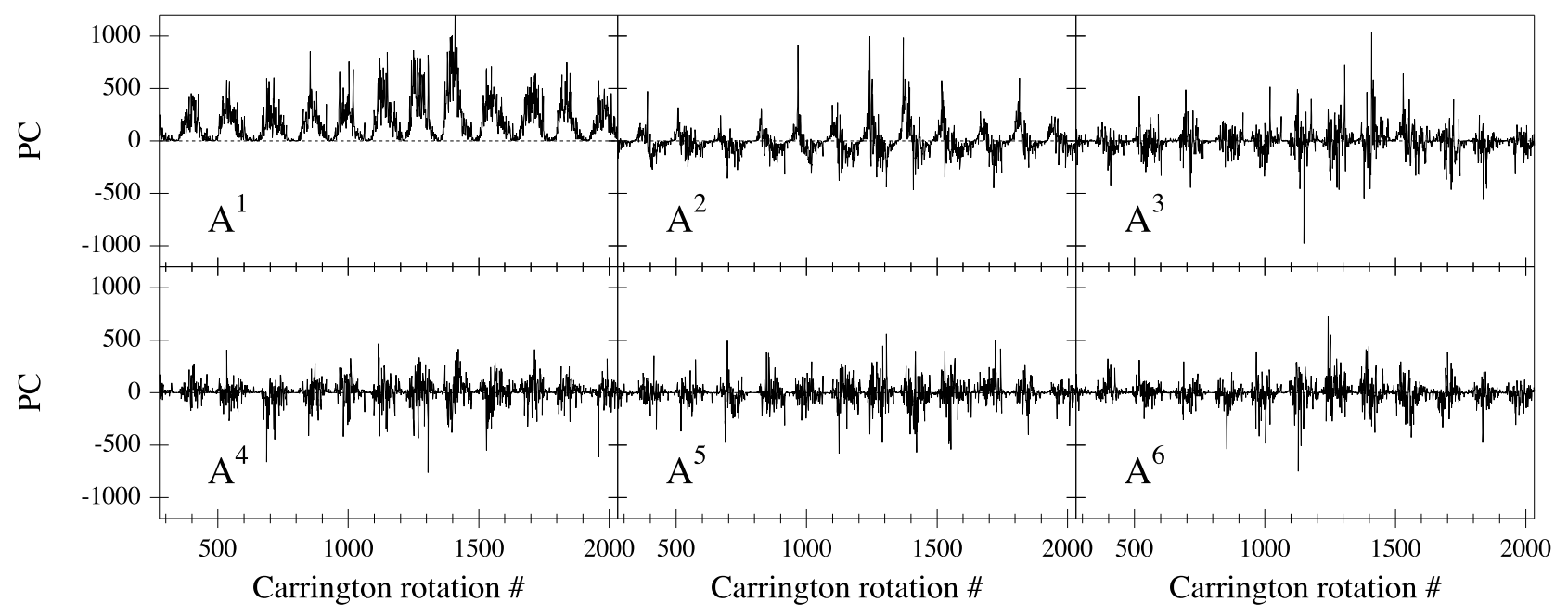

Fig. 4. The first six principal components (PCs) corresponding to the EOFs reported in Fig. 3, and describing the temporal evolution of the EOFs.

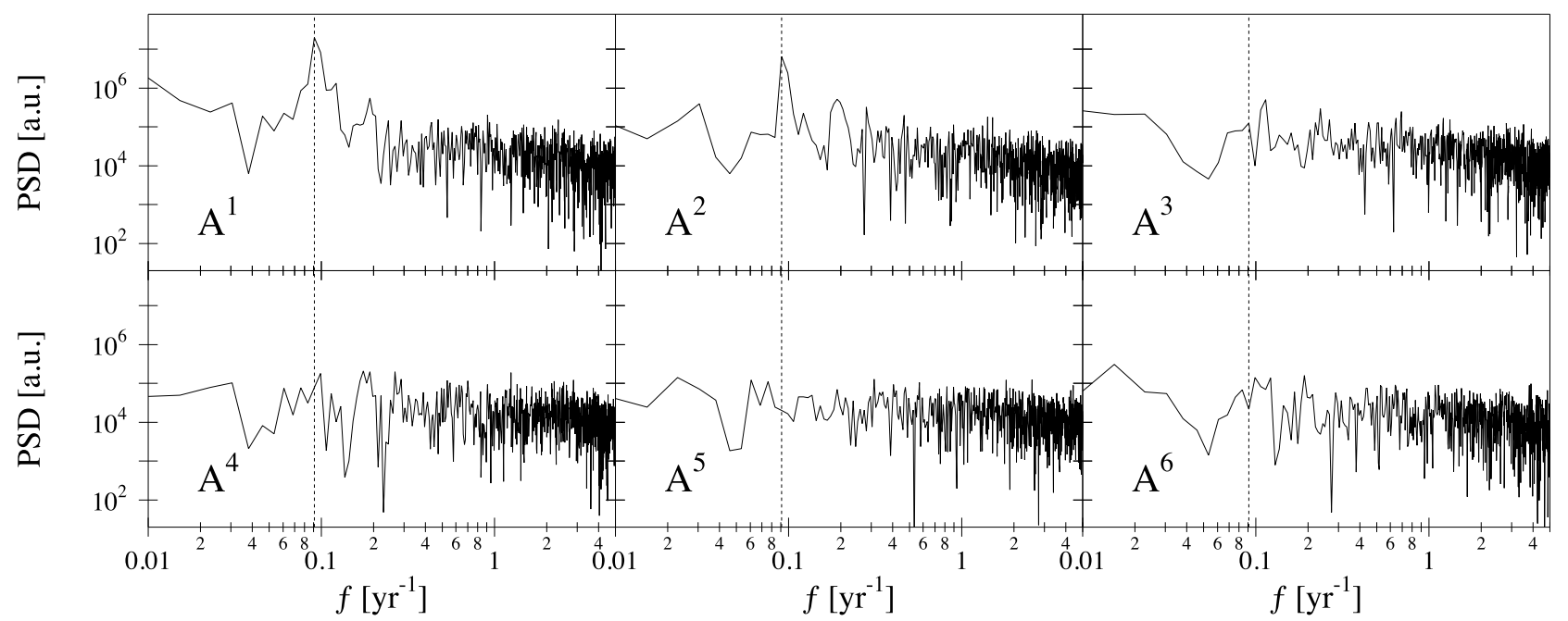

Fig. 5. The Fourier power spectra of the first six PCs. The vertical dotted lines indicate the $10.9 \mathrm{yr}$ characteristic period of solar activity cycle.

Figure 6 shows a reconstruction of the butterfly diagram using only the first two modes. Although the reconstructed diagram qualitatively agrees with the observed behavior, on deeper investigation, we note that the high variability, complexity, and stochasticity observed in the solar cycle are missing. This is clearly evident when the reconstructed diagram is compared to the original one for a single Carrington rotation (see Fig. 7). Evaluating the variability in the solar cycle, we find that the ratio $r_{\sigma}$ of the butterfly diagram variance $\sigma_{o}^{2}$ to that of the reconstructed butterfly $\sigma_{k}^{2}$ with $k=2$ is $\sim 0.4$. This means that the 


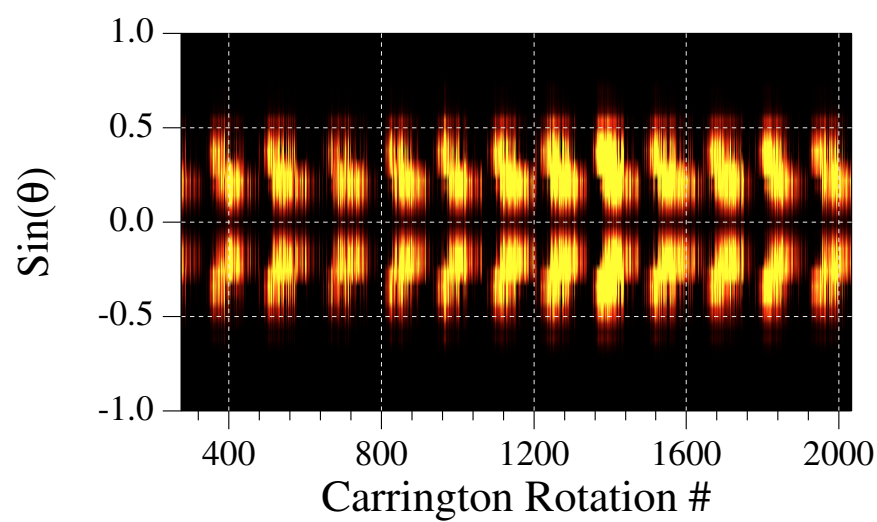

Fig. 6. The butterfly diagram reconstructed according to Eq. (6) using only the first two modes.

first two modes only take into account $40 \%$ of the solar cycle variability.

Since we aim to investigate the chaotic/stochastic features of the solar activity cycle and to discuss them in terms of spatiotemporal and/or dynamical complexity, we must determine the minimum number of modes required to reconstruct the butterfly diagram without losing relevant information about the solar cycle. This can be achieved by studying the average error $\left\langle\delta\left(N^{*}\right)\right\rangle$ as a function of the truncation order $N^{*}$ of the reconstructed butterfly diagram (see Eq. (6)), defined by

$\left\langle\delta\left(N^{*}\right)\right\rangle=\frac{1}{n \times m} \sum_{i, j} \frac{\left|\psi_{N^{*}}\left(x_{i}, t_{j}\right)-\psi\left(x_{i}, t_{j}\right)\right|}{\psi\left(x_{i}, t_{j}\right)}$,

where $\psi_{N^{*}}\left(x_{i}, t_{j}\right)$ is the reconstructed butterfly diagram of truncation order $N^{*}, \psi\left(x_{i}, t_{j}\right)$ is the actual butterfly diagram, $n \times m$ is the total number of points, and the summation over $i$ and $j$ is extended to all the points where $\psi\left(x_{i}, t_{j}\right) \neq 0$. Since the actual butterfly diagram resolution is 1 in units of millionths of a solar hemisphere, we assume that the minimum number $N^{*}$ of modes necessary to reconstruct the butterfly diagram must satisfy the condition $\left\langle\delta\left(N^{*}\right)\right\rangle \leq 1$. In other words, we require that the average truncation error $\left\langle\delta\left(N^{*}\right)\right\rangle$ is less than the data resolution. From the dependence of the truncation error $\left\langle\delta\left(N^{*}\right)\right\rangle$ on truncation or$\operatorname{der} N^{*}$ (Fig. 8), it follows that the minimum number $N^{*}$ of modes necessary to reconstruct the butterfly diagram without losing information is $28 \leq N^{*} \leq 30$. This is why we choose $N^{*}=30$. This choice is also corroborated by the eigenvalue spectrum. Indeed, the first 30 modes contain more than $99.8 \%$ of the true butterfly diagram variance.

\subsection{Chaotic features of PCs}

After having looked at the spectral properties of NOC components and having established the proper number of modes to take into account, we move now to the analysis of the first two PCs.

In Figs. 9 and 10, we display the two plain projections of the phase portrait as reconstructed using $A^{2}(t)$ versus $A^{1}(t)$, and $A^{4}(t)$ versus $A^{3}(t)$, respectively. By smoothing the trajectories over a time window $\sim \frac{1}{4}$ of solar cycle length, it is possible to differentiate between the nature of the first two PCs from the others. While no evidence of a regular temporal structure is found in the $A^{4}(t)$ - $A^{3}(t)$ projection, the smoothed trajectory of $A^{2}(t)$ versus $A^{1}(t)$ exhibits a certain degree of order, as shown by the existence of cyclic oscillations. The spread in the smoothed trajectories in the $\left[A^{2}(t), A^{1}(t)\right]$ plane projection of the phase portrait suggests that

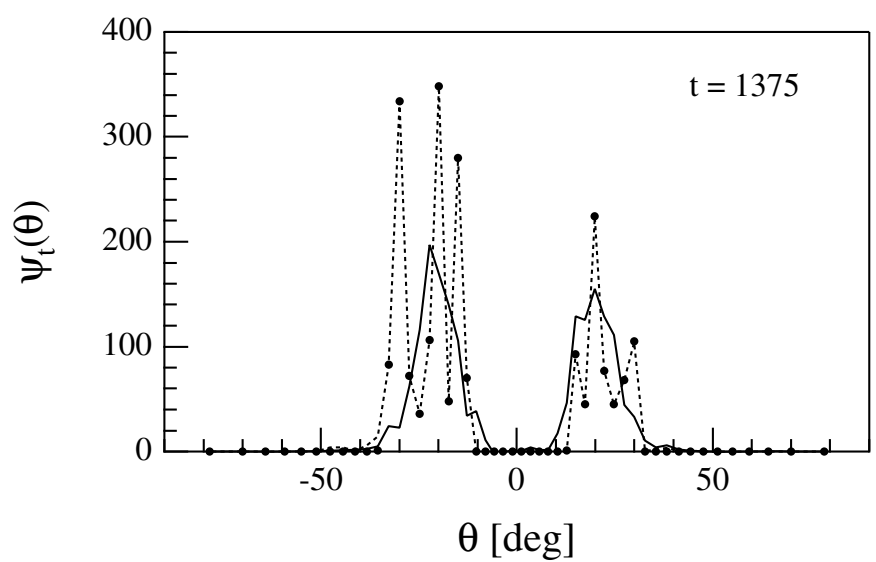

Fig. 7. A comparison between the true sunspot area latitudinal profile (black circles) and the profiles corresponding to the butterfly diagram reconstructed with only two modes (solid line) and with 28 modes (dotted line) for the Carrington rotation $t=1375$. Area is measured in units of millionths of a hemisphere.

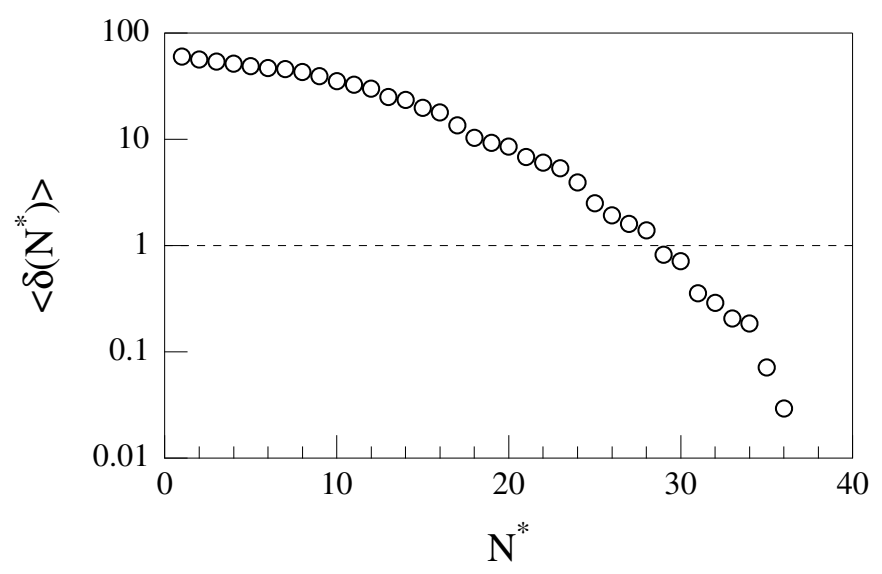

Fig. 8. The average truncation error $\left\langle\delta\left(N^{*}\right)\right\rangle$ as a function of truncation order $N^{*}$. The dotted horizontal line is the threshold corresponding to the butterfly diagram resolution.

chaos and/or stochasticity may play an important role in the dynamics of sunspot cycle. Furthermore, the first two PCs show a phase lag (time shift) of $\sim 2.4 \mathrm{yr}\left(\sim \frac{1}{4}\right.$ of solar cycle) when the cross-correlation function is investigated (data not shown).

To clarify the nature of these sustained oscillations obtained in the case of the smoothed trajectory of $A^{2}(t)$ versus $A^{1}(t)$, we considered a stochastic Lotka-Volterra model (Haken 1983). The Lotka-Volterra (or predator-prey) model describes the dynamics of two coupled variables, which evolves towards a rhythmic behavior for certain initial conditions. The Lotka-Volterra set of equations provides a very simple model for the emergence of sustained oscillations in open systems far from equilibrium (as chemical systems with an overall diverging affinity), as well as for the emergence of spontaneous self-organization (Demirel 2007). Here, we considered a slightly modified version of this simple model, where the coupling between the two variables contains a certain degree of noise. If we indicate by $\left\{n_{i}(t)\right\}$ the set of the time-dependent variables, it follows that

$\left\{\begin{array}{l}\frac{\mathrm{d} n_{1}}{\mathrm{~d} t}=\kappa_{11} n_{1}-\kappa_{12}(t) n_{1} n_{2} \\ \frac{\mathrm{d} n_{2}}{\mathrm{~d} t}=\kappa_{21}(t) n_{2} n_{1}-\kappa_{22} n_{2},\end{array}\right.$

where $\kappa_{11}$ and $\kappa_{22}$ are constants, $\kappa_{12}(t)=\kappa_{21}(t)=\kappa_{\mathrm{c}}(t)=$ $\kappa_{\mathrm{c}}^{0}+\eta(t)$ being $\eta(t)$ a zero-mean delta-correlated Gaussian noise characterized by a variance $\sigma^{2}$, i.e., $\left\langle\eta(t) \eta\left(t^{\prime}\right)\right\rangle_{t}=\sigma^{2} \delta\left(t-t^{\prime}\right)$. 


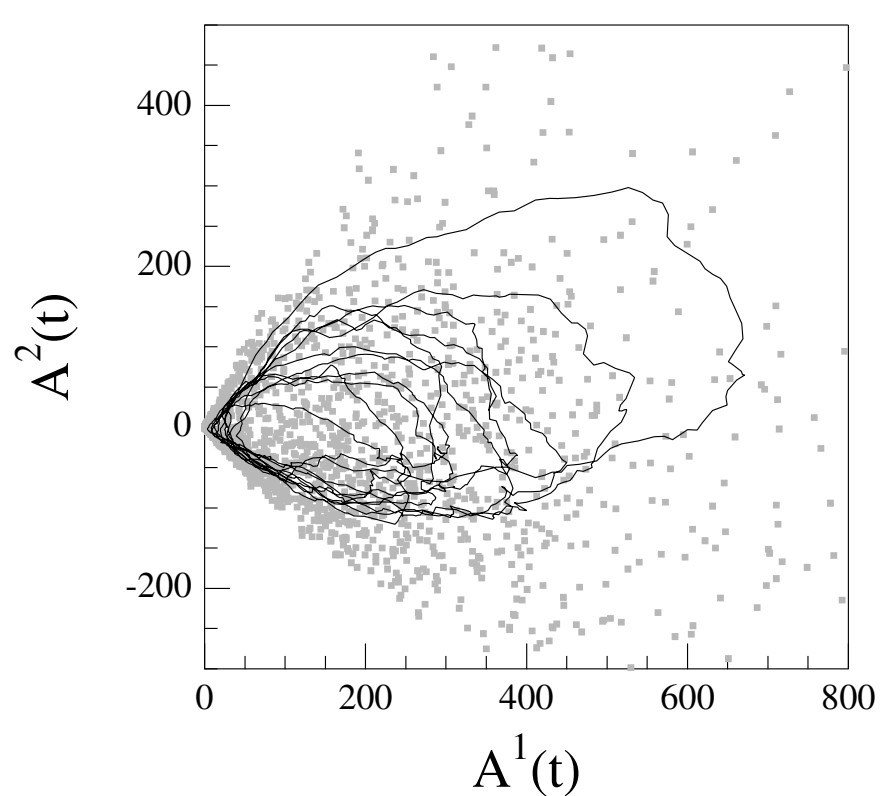

Fig. 9. The 1st plain projection of the unsmoothed phase portrait (grey dots) and the smoothed phase portrait (solid line) reconstructed from the first two PCs. The smoothed version of the phase portrait is obtained using a 37 point ( $\sim \frac{1}{4}$ of solar cycle length) moving window.

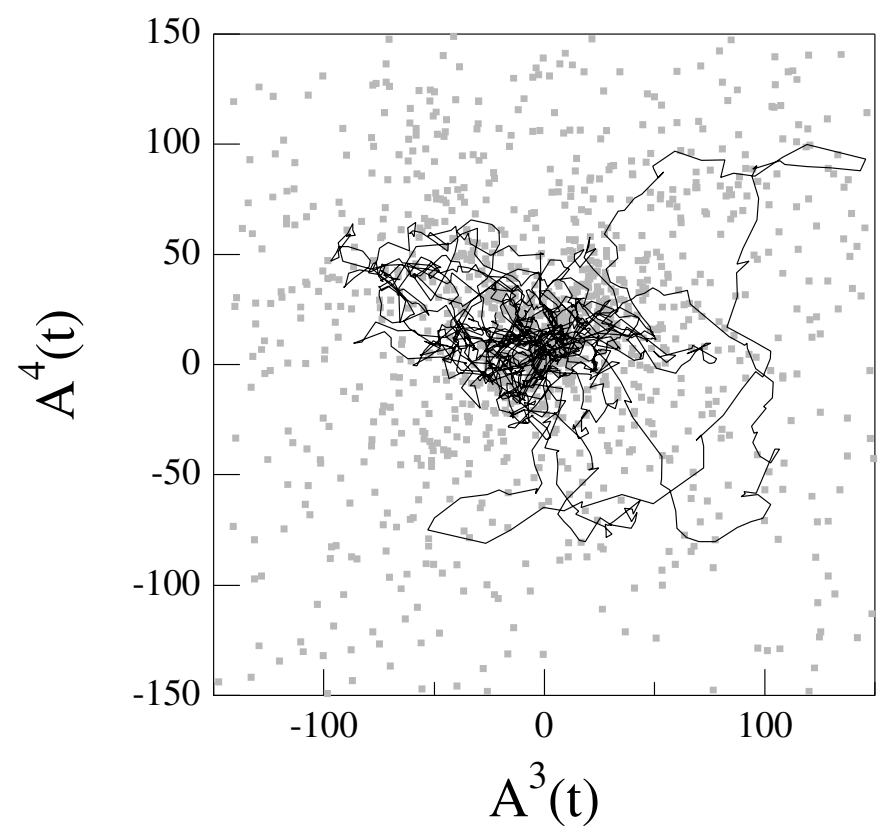

Fig. 10. The 2nd plain projection of the unsmoothed phase portrait (grey dots) and the smoothed phase portrait (solid line) reconstructed from the 3rd and 4th PCs.

Equation (8) was integrated using a 4th-order Runge-Kutta scheme, where the zero-mean delta correlated Gaussian noise was chosen to be characterized by a standard deviation $\sigma=0.2 \%$ of $\kappa_{\mathrm{c}}^{0}$.

The evolution of the two variables $n_{i}$, as resulting from the integration of Eq. (8), has been reported in Fig. 11. With respect to the Lotka-Volterra standard model (see Haken 1983; Demirel 2007, and references therein), the variables show a fluctuating amplitude from one cycle to the other. This behavior resembles that of the solar cycle, whose amplitude changes from one cycle to the other. However, in spite of the stochasticity of the coupling

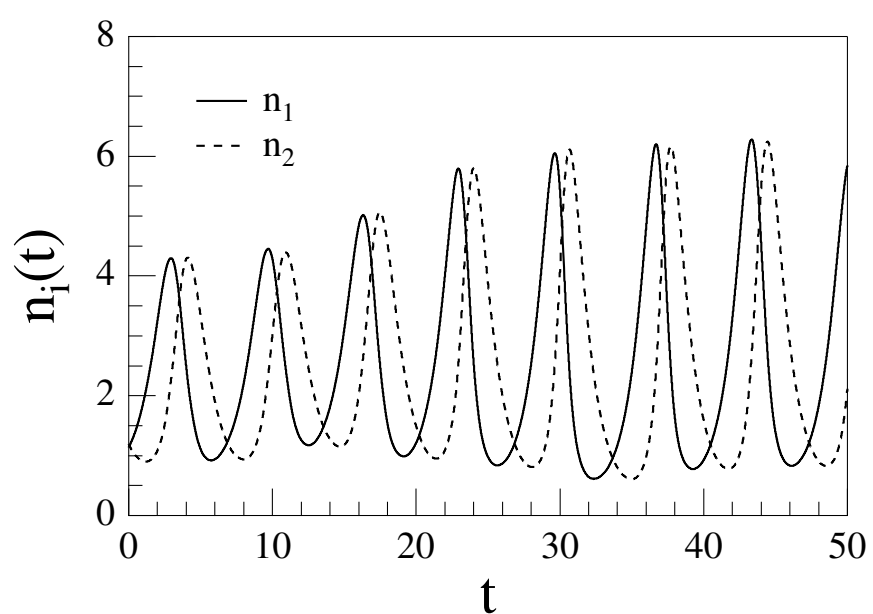

Fig. 11. The behavior of the two variables $\left\{n_{i}\right\}$ of the Lotka-Volterra stochastic model of Eq. (8). Model parameters are $\left(\kappa_{11}, \kappa_{\mathrm{c}}^{0}, \kappa_{22}\right)=$ $(1.0,0.5,1.0)$.

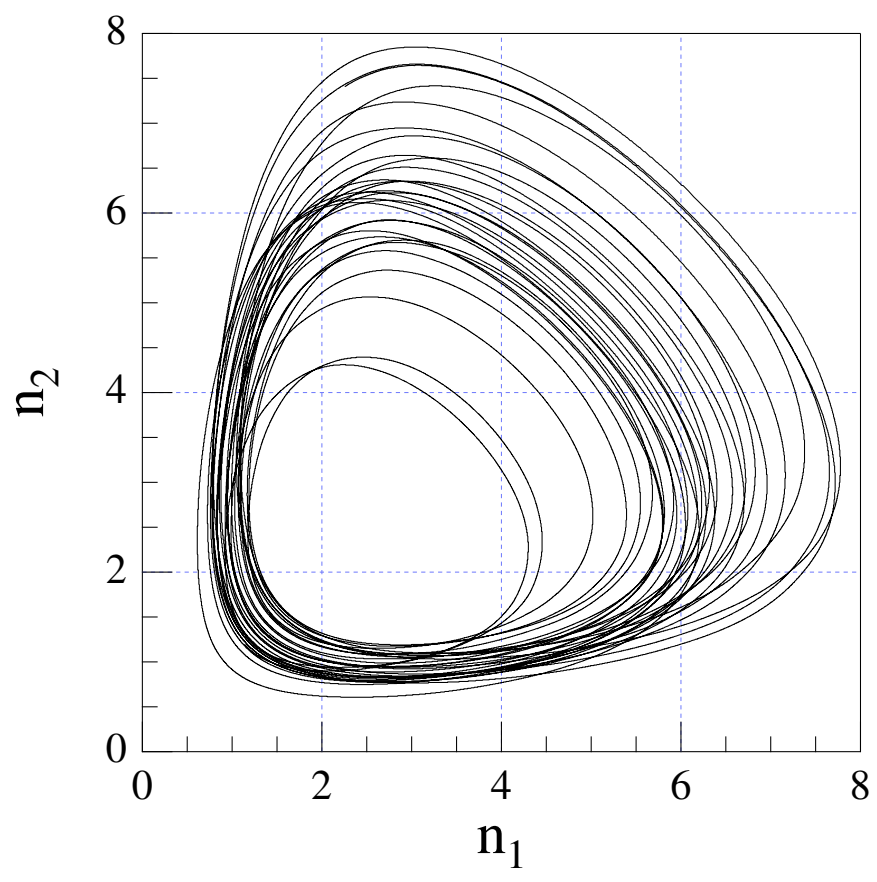

Fig. 12. The $n_{1}-n_{2}$ phase portrait evolution of the solution of Eq. (8).

constant $\kappa_{\mathrm{c}}$, it is still possible to recover an average rhythmic behavior. This is shown in Fig. 12, where we plot the evolution of the stochastic Lotka-Volterra model in the $n_{1}-n_{2}$ plane (phaseportrait).

The parallel with the stochastic Lotka-Volterra model suggests that the main part of the solar cycle variability, described by the first two PCs, can be described in terms of two different sunspot populations, perhaps related to the two modes of the toroidal component of the solar magnetic field (Bigazzi \& Ruzmaikin 2004).

To investigate the relevance of chaos and/or stochasticity to the evolution of the EOFs described by the PCs, as well as of the solar cycle itself, we used the Lyapunov exponents. The Lyapunov exponents are related to the exponential divergence of nearby orbits in the phase space and give both qualitative and quantitative information about the dynamical behavior of the system. In particular, when at least one Lyapunov exponent 
Table 1. Lyapunov exponents of PCs $A^{k}$ up to $k=18$.

\begin{tabular}{cc}
\hline \hline$k$ & $\gamma_{k}$ \\
\hline 1 & $0.14 \pm 0.03$ \\
2 & $0.17 \pm 0.04$ \\
3 & $0.29 \pm 0.04$ \\
4 & $0.30 \pm 0.04$ \\
5 & $0.24 \pm 0.04$ \\
6 & $0.26 \pm 0.04$ \\
7 & $0.38 \pm 0.04$ \\
8 & $0.31 \pm 0.04$ \\
9 & $0.44 \pm 0.04$ \\
10 & $0.36 \pm 0.04$ \\
11 & $0.28 \pm 0.04$ \\
12 & $0.37 \pm 0.04$ \\
13 & $0.36 \pm 0.04$ \\
14 & $0.29 \pm 0.04$ \\
15 & $0.32 \pm 0.04$ \\
16 & $0.40 \pm 0.04$ \\
17 & $0.34 \pm 0.04$ \\
18 & $0.32 \pm 0.04$ \\
\hline
\end{tabular}

is positive, the system is said to be chaotic and small uncertainties in the initial condition can on average increase. For systems whose equations of motion are known, Lyapunov exponents can be estimated quite directly, while their estimation from a finite set of experimental data is a little more complicated. Here, the method proposed by Wolf et al. (1985) was adopted that allows the estimation of the largest positive Lyapunov exponent $\gamma$ if it exists. Starting from the reconstruction of orbits in the phase space, the method is based on monitoring the long-term evolution of the distance between a single pair of orbits.

We estimated the Lyapunov exponents $\gamma$ for the most energetic PCs $A^{k}$ with $k \in[1,30]$. Because of the significant variability in the short timescale fluctuations, we had previously reduced the noise in each PC by applying a simple 13-point moving average, thus filtering out fluctuations below 1 yr. The obtained Lyapunov exponents $\gamma$ are reported in Table 1 up to $k=18$, i.e., to the $k$-value where the exponential decay of the eigenvalue $\lambda_{k}$ spectrum (see Fig. 2) breaks. The first evident result of this analysis is that we can clearly identify two main families of PCs (and EOFs): the first, consisting of the first two PCs, characterized by the same Lyapunov exponent $\langle\gamma\rangle=[0.16 \pm 0.04]$, and the second consisting of all other PCs $(k \in[3,18])$ with an average Lyapunov exponent $\langle\gamma\rangle=[0.32 \pm 0.07]$.

For comparison with the previous analysis of the PCs $\left(A^{k}\right)$, we evaluated the Lyapunov exponent for the numerical solution of the stochastic Lokta-Volterra model, finding for both variables $\gamma_{\mathrm{LV}}=[0.13 \pm 0.03]$, a value very similar to that found for the first two PCs.

As a consequence of the results of the Lyapunov exponent analysis, we can conjecture that the two different families are related to different physical mechanisms. In particular, this analysis supports the idea that the solar cycle cannot be analogous to a self-sustained chaotic cycle (Letellier et al. 2006), but that turbulent (stochastic) fluctuations play a fundamental role in the overall evolution. In other words, the hypothesis that the solar cycle dynamics can be related to low-dimensional chaotic evolution seems to be reductive on the basis of the above results. However, we believe that these noisy fluctuations cannot be assumed to be a stochastic background (Mininni et al. 2002) but instead the result of the evolution of complex diffusive patterns (Lawrence et al. 2005). We return to this point in the following sections.

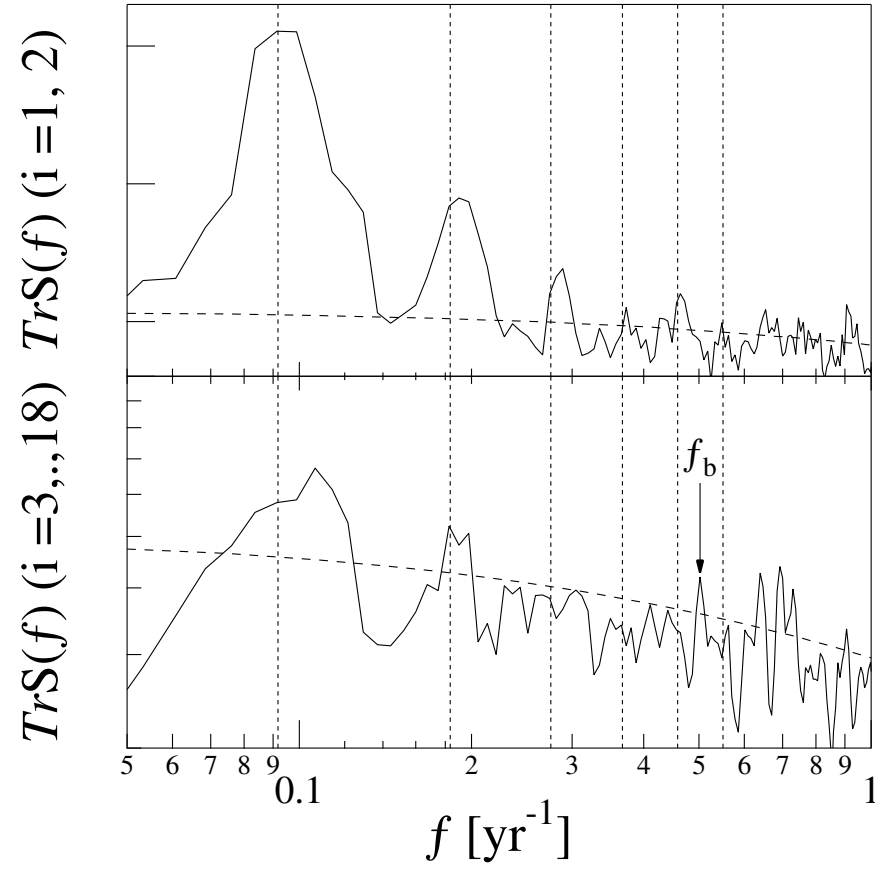

Fig. 13. The trace of the spectral matrix for the two dynamical regimes, $i=1,2$ (upper panel) and $i=[3,18]$ (lower panel), respectively. The dashed lines represent the $95 \%$ confidence levels. Vertical dashed lines refers to the principal solar cycle periodicity and its harmonics (up to 6 ). The arrow in the lower panel indicates a peculiar peak at $\sim \frac{1}{2} \mathrm{yr}^{-1}$, a frequency in good agreement with the well known quasi-biennal oscillation (QBO).

However, to characterize this point, we analyzed the trace of the spectral matrix $S_{i j}(f)$ of the PCs $\left(\operatorname{Tr} \mathbf{S}=\sum_{i} S_{i i}(f)\right)$ for the two different regimes $(i=1,2$ and $i=[3,18])$. The results are reported in Fig. 13. The trace of the spectral matrix for the first dynamical regime is characterized mainly by a pronounced peak at the typical 11-yr periodicity and other peaks close to its harmonics (up to 6), while the trace of the spectral matrix for the second dynamical regime shows a very peculiar peak at $f_{\mathrm{b}} \sim \frac{1}{2} \mathrm{yr}^{-1}$ along with a nearly $11-\mathrm{yr}$ modulation. The observed peculiar periodicity of $T_{\mathrm{b}}=f_{\mathrm{b}}^{-1} \sim 2 \mathrm{yr}$ is in the same range of timescales associated with the quasi-biennial oscillation ( $\sim 2 \mathrm{yr})$, observed in many solar spectral lines and other activity indicators (see e.g., Benevolelenskaya 1998; Polygiannakis et al. 2003; Cadavid et al. 2005; Penza et al. 2006; Laurenza \& Storini 2008).

\subsection{Information entropy and complexity}

To investigate the emergence of spatio-temporal complexity in the solar cycle, we applied some simple concepts of information theory. In particular, moving from a simple parallelism between Eq. (6) and the evolution of states in quantomechanics, it is, indeed, possible to define a time-dependent probability of finding the system in a certain configuration. This is our starting point for the application of information theory. However, we begin by describing the above parallelism in detail.

In quantomechanics, the evolution of a certain state $|\psi\rangle$ can be generally written as

$|\psi\rangle=\sum_{k} c_{k}(t)\left|\phi_{k}\right\rangle$,

where $c_{k}(t)$ is the time-dependent probability amplitude associated with the eigenstate $\left|\phi_{k}\right\rangle$. Since this equation is formally 
equivalent to Eq. (6), we can read the PCs $A^{k}(t)$ as a measure of the probability amplitude of finding the system in the corresponding EOFs $\phi^{k}(x)$. In other words, $A^{k}(t)$ provides a measure of the relative weight of the corresponding $\operatorname{EOF} \phi^{k}(x)$ at the time $t$. Thus, the probability $p_{k}(t)$ associated with $\phi^{k}(x)$ at time $t$ is

$p_{k}(t)=\frac{\left|A^{k}(t)\right|^{2}}{\sum_{j}\left|A^{j}(t)\right|^{2}}$,

where $j \in\left[1, N^{*}\right]$ and $N^{*}$ is assumed to be the truncation order for the reconstruction of the spatio-temporal butterfly diagram according to Eq. (6). On the basis of our discussion in Sect. 3, we assumed $N^{*}=30$.

Figure 14 shows the evolution of the probability $p_{k}(t)$ for the different phases of solar cycle 20 . While in correspondence with the activity maximum, the dominant mode is the first mode, for solar minimum the higher modes $(k>5)$ are more important. This suggests that the solar minimum has a more stochastic character and supports the occurrence of spatio-temporal complexity, which is caused by the competition between stochastic fluctuations and regular evolution.

To characterize the long term evolution of the spatiotemporal complexity in the solar cycle with greater accuracy, we move from the previous definition of a probability spectrum and apply the concepts of information theory to investigate the degree of stochasticity in the solar cycle evolution. We evaluate the Shannon's information entropy $S(t)$ (Shannon 1948) defined to be

$S(t)=-k_{S} \sum_{k=1}^{N^{*}} p_{k}(t) \ln p_{k}(t)$,

where $k_{S}$ is a constant assuming the role of the Boltzmann's constant that we have here assumed to be $k_{S}=1$ for convenience. The entropy $S(t)$, named also unconditional entropy or Boltzman-Gibbs-Shannon entropy, provides a measure of the information/uncertainty content in the probability spectrum, and is within the interval $S \in\left[0, \ln \left(N^{*}\right)\right]$. Since the higher the value, the wider the spectrum of accessible states, $S(t)$ provides a measure of disorder (uncertainty). If the energy is confined in only one mode, $S$ will be exactly zero, i.e., we are in a state of maximum order. In contrast, for a uniform distribution $\left(p_{k}=1 / N^{*}\right)$ we obtain $S=\ln \left(N^{*}\right)$ corresponding to the minimum information (maximum uncertainty).

Figure 15 shows the evolution of information entropy $S(t)$ for the solar activity cycle evaluated using Eqs. (10) and (11), compared with the solar cycle as measured by the sunspot hemispheric area coverage. Although $S(t)$ is strongly variable (its average value $\langle S(t)\rangle_{t}$ is $\sim 2$ ), the temporal behavior of the information entropy exhibits a certain degree of anticorrelation with the solar activity cycle. The information entropy is generally higher during solar minima than during maxima, reflecting the more noisy structure of the butterfly diagram at solar minima. This anticorrelation is confirmed by the Pearson linear correlation coefficient ( $r=-0.44$ ) between the smoothed $S(t)$ and the sunspot coverage $A_{T}$. Figure 16 shows the power spectral density (PSD) of the information entropy $S(t)$. The PSD has a more complex structure with respect to the single PCs, showing many maxima emerging from the nearly-flat background of the characteristic solar cycle periodicity and its harmonics. However, the nearlyflat background $\left(\sim f^{-\eta}\right.$ with $\left.\eta \sim 0.3\right)$ suggests that the stochastic fluctuations play a relevant role in the spatio-temporal complexity displayed by the solar activity cycle.

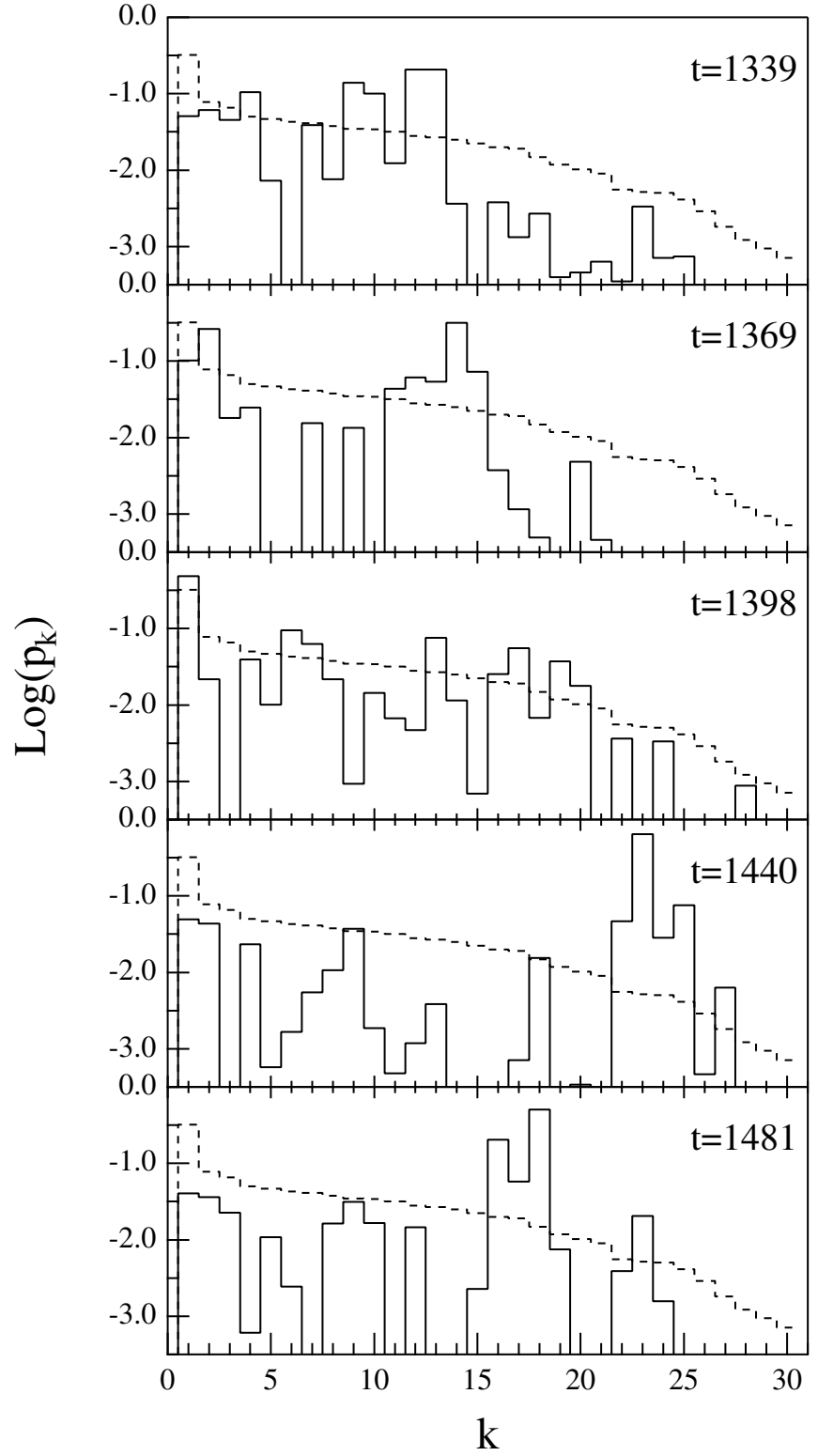

Fig. 14. Evolution of the probability $p_{k}(t)$ (plotted as $\log \left[p_{k}(t)\right]$ ) for the solar cycle 20. From top to bottom, solar minimum $(t=1339)$, ascending phase $(t=1369)$, maximum $(t=1398)$, descending phase $(t=1440)$, and minimum $(t=1481)$. The solid lines refer to the instantaneous probability spectrum, while the dashed lines indicate the average spectrum. Time $t$ is measured in Carrington rotations.

Although the information entropy is a useful quantity to provide insights into the complex dynamics of a many degree-offreedom system, a more accurate characterization of the spatiotemporal complexity observed in a dynamical system could be given by the II order complexity measure $\Gamma_{11}$ introduced by Shiner et al. (1999; see also Crutchfield et al. 2000; Binder \& Perry 2000; and Shiner et al. 2000). From the definition of the information entropy $S(t)$, it is possible to define a disorder measure $\Delta(t)$ (Landsberg 1984) by simply normalizing the information entropy to the maximum entropy $S_{\max }$, i.e.,

$\Delta(t)=\frac{S(t)}{S_{\max }}$.

The disorder measure $\Delta$ is limited to the interval $[0,1]$, where $\Delta \rightarrow 0(\Delta \rightarrow 1)$ indicates a higher degree of order (disorder). 


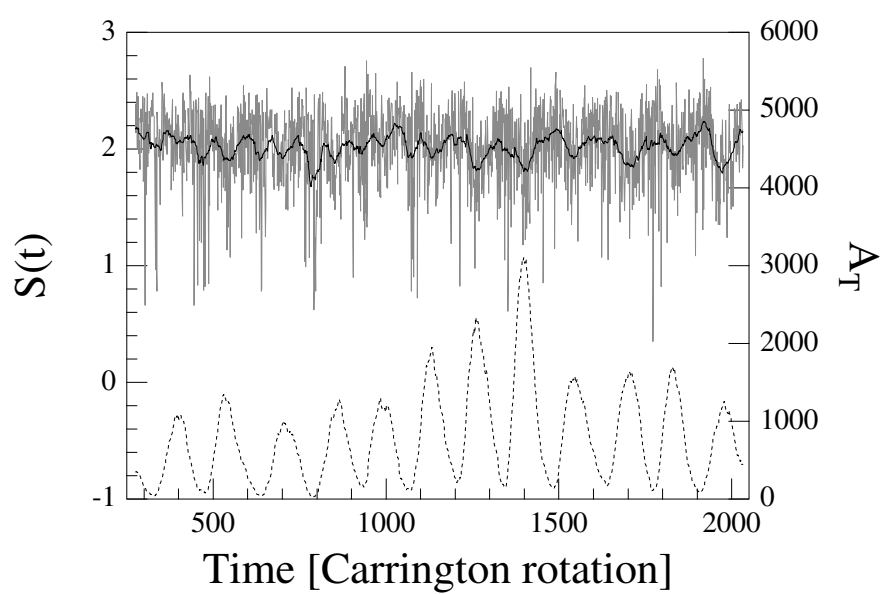

Fig. 15. The evolution of the information entropy $S(t)$ for the solar activity cycle evaluated using Eq. (11) in comparison with the solar activity cycle measured by the sunspot hemispheric area coverage (dotted line). The solid black line is a smoothed version of $S(t)$ using a moving window of 37 Carrington rotations (i.e., $~ 1 / 4$ of the solar cycle periodicity).

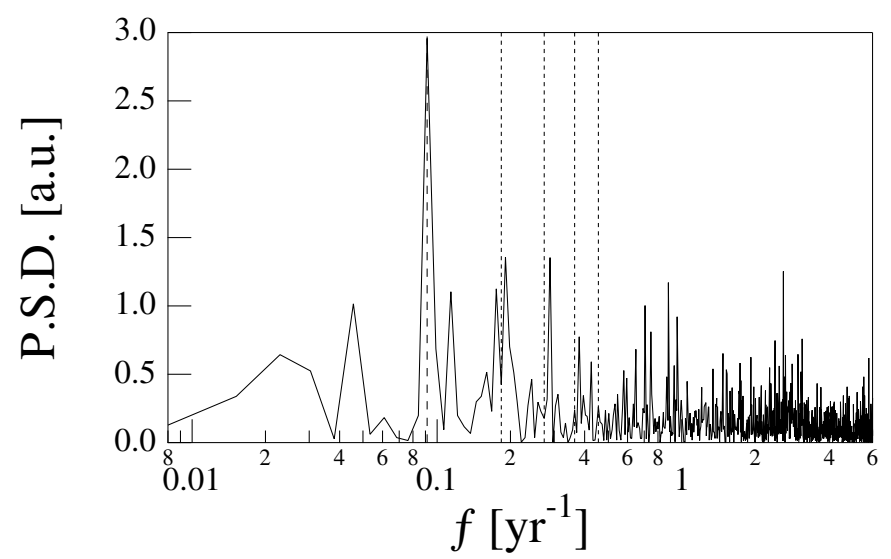

Fig. 16. The power spectral density (PSD) of the information entropy $S(t)$. The dashed vertical line indicates the solar cycle characteristic period $\left(T_{0} \sim 10.9 \mathrm{yr}\right)$, while the dotted vertical lines correspond to the harmonics of the solar cycle characteristic period $\left(T_{n}=T_{0} / n\right.$ with $n=2,3, \ldots, 5)$.

According to Shiner et al. (1999), it is possible to define a generalized II order complexity measure $\Gamma_{\alpha \beta}$ to be

$\Gamma_{\alpha \beta}=\Delta^{\alpha}(1-\Delta)^{\beta}$,

among which a special role is played by the $\Gamma_{11}$ complexity measure that is equal to $\Gamma_{11}=\Delta(1-\Delta)$. If we remember that the equilibrium configuration generally corresponds to a maximum entropy state $S_{\max } \equiv S_{\text {eq }}$, the complexity measure $\Gamma_{11}$ will vanish at both equilibrium and complete order, implying that complexity will increase in intermediate situations. For instance, in nonequilibrium open systems the emergence of coherent features and structures can be associated with a reduction in the entropy content, i.e., with an increase in the degree of order (Klimontovich 1991). Another important feature of the aforementioned approach to complexity is that the $\Gamma_{11}$ measure can be applied to systems with a varying number of degrees-offreedom.

The first step in estimating the $\Gamma_{11}$ complexity measure is the choice of the reference maximum entropy $S_{\max }$. As this is a subtle point, we provide a careful discussion of this choice.

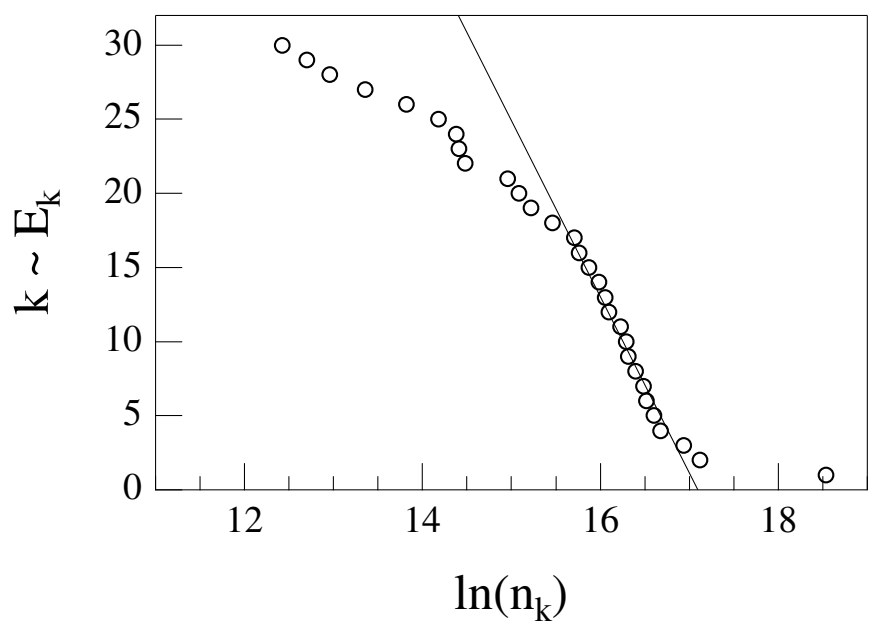

Fig. 17. The order $k\left(\sim E_{k}\right)$ versus the natural logarithm of the occupation number $n_{k}$. The solid line is a linear plot defining the effective temperature $T_{\text {eff }}$.

From the definition of the Shannon's information (see Eq. (11)), it follows that the maximum entropy is attained by considering an equiprobability situation. Thus, the simplest assumption for the maximum entropy would be that $S_{\max } \propto \ln \left(N^{*}\right)$, where $p_{k}=1 / N^{*}$. This situation corresponds to the case of an isolated (microcanonical) system based on the assumption, as a postulate, of a uniform probability for all the microstates corresponding to the same macrostate (Ben-Naim 2008). Although this is a reasonable hypothesis, its applicability to the solar cycle dynamics is questionable. In the case of solar cycle dynamics, we deal with a nearly stationary nonequilibrium state, where the assumption of equiprobability for the occupation number (state amplitude) could lead to an overestimation of the reference maximum entropy. This point is also corroborated by the assumption of Shiner et al. (1999) of assuming $S_{\text {eq }}$ to be the reference maximum entropy. In other words, we have to find a way of defining a reasonable reference stationary distribution $\hat{p}_{k}$ to be able to evaluate the reference maximum entropy.

By looking at the eigenvalue $\lambda_{k}$ spectrum (see Fig. 2), we can immediately realize that it is possible to define a reference $\hat{p}_{k}$ distribution by again using our parallelism with quantomechanics. By assuming that $\lambda_{k}$ is equivalent to the occupation number in the state $|k\rangle$ of energy $E_{k} \propto k$, it is indeed possible to introduce an effective temperature $T_{\text {eff }}$ and a reference nearly exponential distribution $\hat{p}_{k}$ for the reference stationary state. Figure 17 shows $k \propto E_{k}$ versus the natural logarithm of the occupation number $n_{k} \equiv \lambda_{k}$. A linear region can be found in the interval range $k \in[3,18]$, from which an effective temperature $T_{\text {eff }}$ (data not shown) can be estimated to be

$\lambda_{k} \sim \exp \left(-\frac{k}{T_{\text {eff }}}\right)$

Thus, for the reference distribution $\hat{p}_{k}$ we can assume that

$\hat{p}_{k}=\frac{\exp \left(-k / T_{\text {eff }}\right)}{\sum_{j=1}^{N^{*}} \exp \left(-j / T_{\text {eff }}\right)}$,

where $N^{*}=30$. From the reference distribution $\hat{p}_{k}$, we can derive $S_{\max }=3.182<\ln (30)$ for the reference maximum entropy, and consequently according to Eqs. (12) and (13), we obtain the corresponding disorder measure $\Delta$ and the II order complexity measure $\Gamma_{11}=\Delta(1-\Delta)$. 


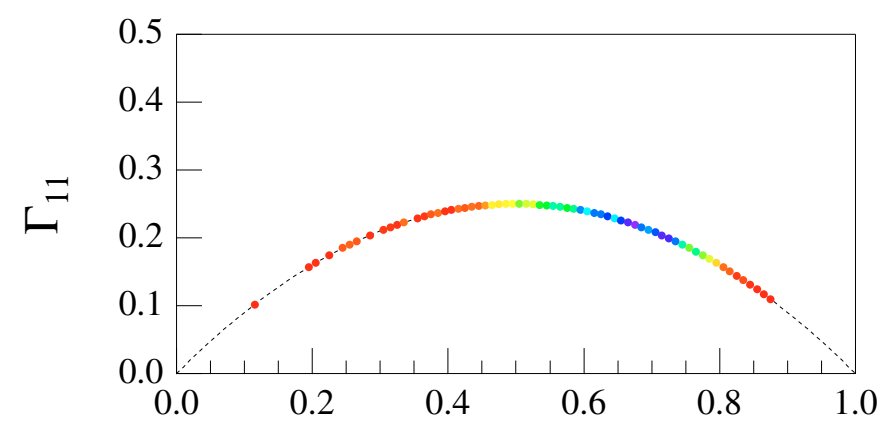

$\Delta$

Fig. 18. The II order complexity measure $\Gamma_{11}$ versus disorder $\Delta$ for the solar cycle. The colors (from red to violet) are an increasing measure of the probability of the occurrence of $\Delta$ in the solar cycle. Note that the maximum probability (violet dots) corresponds to $\Delta \sim 0.68$ and $\Gamma_{11} \sim 0.22$.

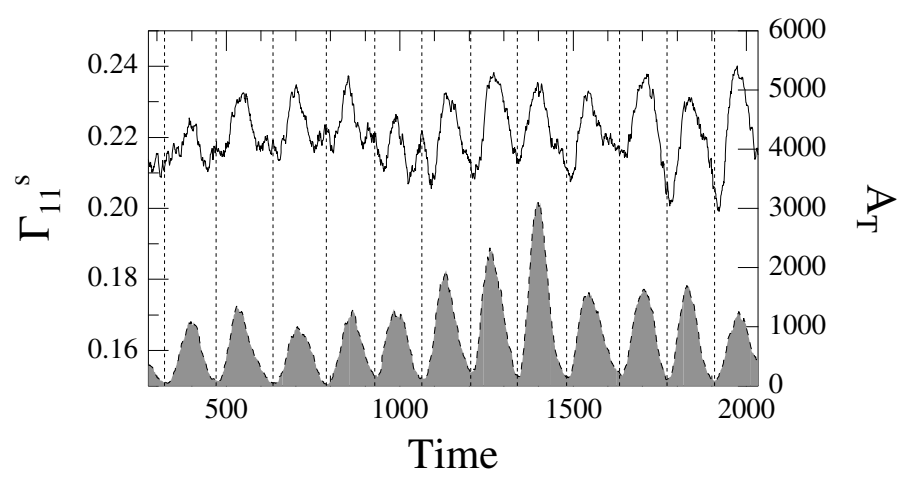

Fig. 19. A comparison between the II order complexity measure $\Gamma_{11}^{s}$ (left axis - solid line), averaged over 37 Carrington' solar rotations, and the solar cycle measured in terms of the total hemispheric sunspot coverage $A_{T}$ (right axis - dashed line and gray area). Note that $\Gamma_{11}^{s}$ complexity reaches a maximum for the solar maxima ( $\Delta$ is minimum), while the minima of the solar cycle correspond to more disordered and less complex configurations $\left(\Delta \rightarrow 1\right.$ and $\left.\Gamma_{11}^{s} \rightarrow 0\right)$.

Figure 18 shows the behavior of $\Gamma_{11}$ as a function of disorder $\Delta$ for the solar cycle. We note that the most probable value of $\Gamma_{11}$ is $\sim 0.22$ in correspondence to $\Delta \sim 0.68$, a value that clearly underlines how complexity plays a fundamental role in the evolution of solar cycle. To associate complexity with the different phases of the solar cycle more accurately in Fig. 19 we compare the complexity measure $\Gamma_{11}^{s}$, averaged over 37 Carrington solar rotations to reduce small-scale noise (see also Fig. 15) and emphasize its mid-long term evolution with the corresponding solar cycle, described by the total hemispheric sunspot coverage $\left(A_{T}\right)$. Solar maxima correspond to complex states, while solar minima relate to more disordered and less complex configurations. This result, inferred from the existence of two different dynamical regimes/processes (as shown in previous analyses), clearly suggests that the sunspot cycle is the result of interacting processes in an open system.

\section{Summary and conclusions}

We have focused our present study on the emergence of dynamical (spatio-temporal) complexity in the 11-yr solar cycle as monitored by the sunspot activity, by applying non-traditional approaches and techniques based on information theory. Although the global characteristics of the solar cycle are described well by dynamo theory, the origin of its complex irregularities remains unclear.

We decomposed the space-time distribution of sunspot areas over 1759 Carrington rotations by applying the natural orthogonal composition technique, whose primary characteristics is the identification of the most suitable set of basis orthogonal functions (the empirical orthogonal functions $-\phi^{k}$ ). Results obtained by using this technique confirm previous findings of a $T_{1} \sim 11 \mathrm{yr}$ periodicity (found in the first two PCs), which characterizes the long-term evolution of the solar cycle. A less prominent periodicity was found to correspond to the second harmonic $\left(T_{2} \sim 5.5 \mathrm{yr}\right)$. However, while the periodicity at $T_{1}$ agrees with similar results for the solar magnetic cycle (Mininni et al. 2002, 2004; Lawrence et al. 2005), the one at $T_{2}$ emerges only after studying the 11-year cycle.

Apart from the characteristic spectral features of the PCs, an interesting point about the NOC results is that the main part of the 11-yr solar activity cycle can be described by two components (the first two EOFs and the associated PCs) shifted in time by $\sim 2.4$ yr $\left(\sim \frac{\pi}{2} \equiv \frac{1}{4}\right.$ of solar cycle) with respect to each other. The observed shift in time is in agreement with the phase shift $\left(\sim \frac{\pi}{4}\right)$ between the $m=0$ and $m=1$ modes of the toroidal components of the magnetic field as observed in numerical simulations of non-axisymmetric dynamo model with mean helicity $\alpha$, located mostly above the tachocline (Bigazzi \& Ruzmaikin 2004), and taking into account that solar magnetic cycle periodicity is expected to be twice that of the sunspot cycle. By inspecting the shape of the first two EOFs, and in particular the latitudinal position of their peaks, we note how the second EOF is mainly responsible for the high latitude increase in the number of sunspot structures observed during the early stages of the solar cycle, while the first EOF describes mainly the nearly symmetric low latitude structures. This result suggests that the solar activity cycle can be described by two main interacting modes, whose character resembles the behavior of a simple stochastic two populations Lotka-Volterra model. Again, the spatial distribution of these first two EOFs, which are peaked at about $\left(10^{\circ} ; 20^{\circ}\right)$ and $\left(20^{\circ} ; 30^{\circ}\right)$ latitude, resembles that of the first two modes of the field toroidal component, which were found to be located at differently latitudes (Bigazzi \& Ruzmaikin 2004). We believe that these two different fundamental modes, as monitored by the first two EOFs, are manifestations of the first two modes of the toroidal magnetic field component. We note that the observed time delay of $\sim 2.4 \mathrm{yr}$ corresponds to the same range of timescales characteristic of the so-called Gnevyshev gap (Storini et al. 2004). This observation and since the second EOF mainly describes high latitude structures and the second PC precedes the first, is in close agreement with the pioneering observations of Gnevyshev $(1963,1977)$, providing a possible origin of the observed gap.

Another interesting result obtained from the NOC analysis is the dual character of the eigenvalue spectrum, which shows the existence of two different regimes. This result, together with the two corresponding families identified by the Lyapunov's exponents $\gamma$, allows us to conjecture that the solar cycle may be analogous to a self-sustained chaotic cycle plus a certain amount of turbulent fluctuations.

Indeed, it has been shown (Hoyng et al. 1994) that in a simple axisymmetric mean-field dynamo, random $\alpha$-fluctuations can account for several features of the observed solar cycle. In particular, these random fluctuations, expected to arise from the turbulent convection, are characterized by a correlation time much shorter than any characteristic dynamo period. We believe that 
the second regime in the eigenvalue spectrum could be a manifestation of these random fluctuations in the $\alpha$-effect. This hypothesis is supported by the values of the Lyapunov exponents, which are higher in the second regime than the first, therefore implying a higher variability.

We note that the presence of two distinct dynamical regimes with different temporal (spectral) properties supports the hypothesis suggested by Benevolenskaya $(1998,2000)$ of a double cycle characterized by different timescales. Benevolenskaya $(1998,2000)$ suggested a double (dynamo) mechanism for the solar magnetic cycle, the first generated at the base of the convection zone by large-scale radial shear of the angular velocity and responsible for the 22-yr Hale's cycle and, thus, for the usual 11-yr activity cycle (Ruzmaikin 1996); the second due to latitudinal or radial shears in the subsurface regions. Although evidence of these two double mechanisms has been found, the 11-yr modulation in the spectrum of the second regime suggests that at the origin of the observed complexity there is a nonlinear coupling between the mechanisms.

The results obtained from the application of the information theory approach to the solar cycle have emphasized the appearance of dynamical complexity and indicated how complexity increases during the maximum phases of the solar cycle. In particular, we have found that, in correspondence to the solar maximum, the first two mode dominate over the others, while towards solar minimum the higher modes become more important. As already suggested by Mininni et al. (2002), this could be interpreted in terms of a higher level of stochasticity of the solar minimum competing with the regular evolution characteristic of the solar maximum. However, the degree of stochasticity observed during the solar minima never equals the maximum value $(\Delta(t)<1 \forall t)$. This point supports the idea that the higher modes could be responsible for the evolution of complex diffuse field patterns as proposed by Lawrence et al. (2005). A quantitative estimation of the solar cycle complexity by a II order complexity measure (Shiner et al. 1999) confirmed the above scenario $\left(\Delta \sim 0.68\right.$ and $\left.\Gamma_{11} \sim 0.22\right)$. On the basis of this result, the spatio-temporal variability originating in the higher order modes could be the outcome of a superficial turbulent dynamo process (i.e., of a highly complex phenomenon) in the sunspot evolution and dynamics. Consequently, the emergence of dynamical complexity excludes the hypothesis that the solar cycle variability is caused by low-dimensional chaos (Letellier et al. 2006) or pure stochastic dynamics. However, we propose that the observations of low-dimensional chaos in the sunspot cycle (Letellier et al. 2006) could be the consequence of the use of coarse-grained descriptors (such as the Wolf's sunspot number), which are unable to convey all the information contained in spatio-temporal diagrams such as the butterfly diagram. By limiting our attention to the first two modes (PCs), the dynamics studied was found to agree with nearly low-dimensional chaotic behavior, as emphasized by comparison with the stochastic Lotka-Volterra model. Moreover, although in the past few solar cycles the fluctuations in the information entropy $S(t)$ have increased in amplitude, we have not found clear evidence of an increasing trend in this quantity with time. Thus, we cannot confirm the previous findings by Sello (2000) of an increase in disorder from cycle 23rd onwards. We believe that this difference could be caused mainly by the different datasets and the different analysis techniques (wavelets versus NOC). We also note that the last cycles are characterized by larger fluctuations in the complexity measure.
In summary, we have illustrated the complex nature of the solar cycle and pointed out that this complexity is probably caused by the nonlinear coupling of different processes.

Acknowledgements. The Authors thank F. Berrilli, M. Laurenza, V. Penza and M. Materassi for valuable discussions and comments on this work. We acknowledge the NASA/Solar Physics Marshall Space Flight Center (http:// Science.nasa.gov/ssl/pad/solar/greenwch.htm) for making available the data used in this work.

\section{References}

Badii, R., \& Politi, A. 1997, Complexity: hierarchical structures and scaling in physics (Cambridge, UK: Cambridge University Press)

Ben-Naim, A. 2008, A farewell to entropy: statistical thermodynamics based on information (Singapore: World Scientific Publishing Co. Pte. Ltd.)

Benevolelenskaya, E. E. 1998, ApJ, 509, L49

Benevolelenskaya, E. E. 2000, Sol. Phys., 191, 247

Bigazzi, A., \& Ruzmaikin, A 2004, ApJ, 604, 944

Binder, P.-M., \& Perry, N. 2000, Phys. Rev. E, 62, 2998

Cadavid, A. C., Lawrence, J. K., McDonald, D. P., \& Ruzmaikin, A. 2005, Sol. Phys., 226, 359

Chang, T., Tam, S.W.Y., \& Wu, C.-C. 2006, Space Sci. Rev., 122, 281

Crutchfield, J. P., Feldman, D. P., \& Rohilla Shalizi, C. 2000, Phys. Rev. E, 62, 2996

Demirel, Y. 2007, Nonequilibrium thermodynamics, Transport and rate processes in physical, chemical and biological systems, 2nd edition (Amsterdam: Elsevier)

Golovkov, V. P., Papitashvili, N. E., Tyupkin, Y. S., \& Knarin, E. P. 1978, Geomag. \& Aeron., 18, 511

Golovkov, V. P., Papitashvili, V. O., \& Papitashvili, N. E. 1989, Geomag. \& Aeron., 29, 514

Golovkov, V. P., Kozhoyeva, K. G., \& Shkolǹikova, S. I. 1992, Geomag. \& Aeron. 32, 715

Gnevyshev, M. N. 1963, Soviet Astron.-AJ, 7, 311

Gnevyshev, M. N. 1977, Sol. Phys., 51, 175

Haken, H. 1983, Synergetics, an Introduction: Nonequilibrium Phase Transitions and Self-Organization in Physics, Chemistry, and Biology (New York: Springer-Verlag)

Hoyng, P., Schmitt, D., \& Teuben, L. J. W. 1994, A\&A, 289, 265

Jackson, G. M., Mason, I. M., \& Greenhalgh, S. A. 1991, Geomag. \& Aeron., 56,528

Klimontovich, Yu. L. 1991, Turbulent Motion and the Structure of Chaos, A New Approach to the Statistical Theory of Open Systems (Dordrecht: Kluwer Academic Publishers)

Klimontovich, Yu. L. 1996, Phys. Uspekhi, 39, 1169

Landsberg, P. T. 1984, Phys. Lett., 102A, 171

Laurenza, M., \& Storini, M. 2008, Mem. S.A.It., in press

Lawrence, J. K., Cadavid, C., \& Ruzmaikin, A. 2005, Sol. Phys., 225, 1

Letellier, C., Aguirre, L. A., Maquet, J., et al. 2006, A\&A, 449, 379

Mininni, P. D., Gomez, D. O., \& Mindlin, G. B. 2002, Phys. Rev. Lett., 89, 061101

Mininni, P. D., López Fuentes, M., Mandrini, C. H., et al. 2004, Sol. Phys., 219, 367

Nicolis, G., \& Nicolis, C. 2007, Foundations of Complex Systems. Nonlinear Dynamics, Statistical Physics, Information and Prediction (Singapore: World Scientific Publishing Co. Pte. Ltd.)

Penza, V., Pietropaolo, E., \& Livingston, W. 2006, A\&A, 454, 349

Polygiannakis, J., Preka-Papadema, P., \& Moussas, X. 2003, MNRAS, 343, 725 Rotanova, N. M., Papitashvili, N. Ye., \& Pushkov, A. N. 1982, Geomag. \& Aeron., 22, 821

Ruzmaikin, A. A. 1996, Geophys. Res. Lett., 23, 2649

Shannon, C. E. 1948, Bell Syst. Tech. J., 27, 379

Shiner, J. S., Davison, M., \& Landsberg, P. T. 1999, Phys. Rev. E, 59, 1459

Shiner, J. S., Davison, M., \& Landsberg, P. T. 2000, Phys. Rev. E, 62, 3000

Sello, S. 2000, A\&A, 363, 311

Sokoloff, D., \& Nesme-Ribes, E. 1994, A\&A, 288, 293

Storini M., et al. 2004, Adv. Space Res., 31, 895

Vecchio, A., Primavera, L., Carbone, V, et al. 2005, Sol. Phys., 229, 359

Xu, W.-Y. 2003, Geophys. J. Inter., 152, 613

Xu, W.-Y., \& Kamide, Y. 2004, J.G.R., 109, A05218

Wolf, A., Swift, J. B., Swinney, H. L., et al. 1985, Physica D, 16, 285 